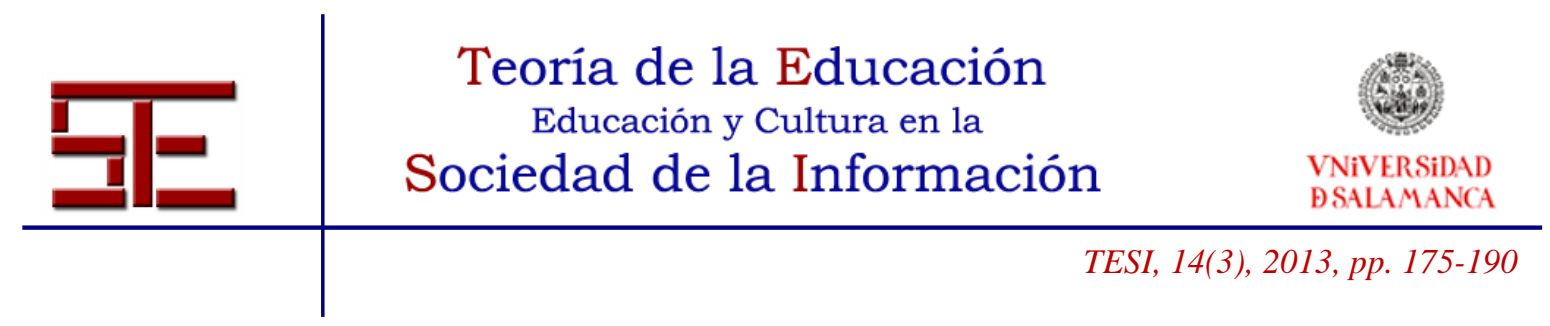

\title{
ENTORNOS HIPERTEXTUALES Y EDUCACIÓN
}

Resumen: En el presente trabajo pretendemos analizar, de forma sintética, el marco desde el que la educación formal y sus principales actores (estudiantes y docentes) deberían contemplar los entornos hipertextuales o, si se quiere, la hipertextualidad, entendiéndola no sólo como una herramienta específica de comunicación y de aprendizaje sino, también, como algo que se inscribe en el marco de los procesos mentales-cognitivos y, aún de forma más amplia, en los contextos actitudinal y filosófico de cualquier proceso pedagógico.

Palabras clave: Hipertexto, cognición, educación, docente.

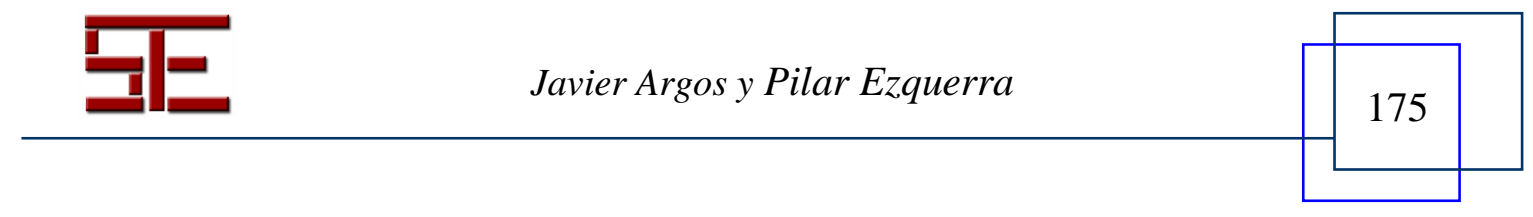




\section{HYPERTEXT ENVIRONMENTS AND EDUCATION}

Abstract: this paper aims to in brief analyze the framework from which formal education and its main actors (students and teachers) should provide hypertext environments, that is, the hypertextual domain. Such environments are understood not only as a specific communication and learning instruments, but also as something that falls within the category of mental-cognitive processes and, even more broadly, attitudinal and philosophical contexts of any educational process.

Key words: Hypertext; cognition; education; teacher.

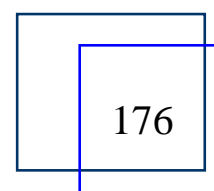




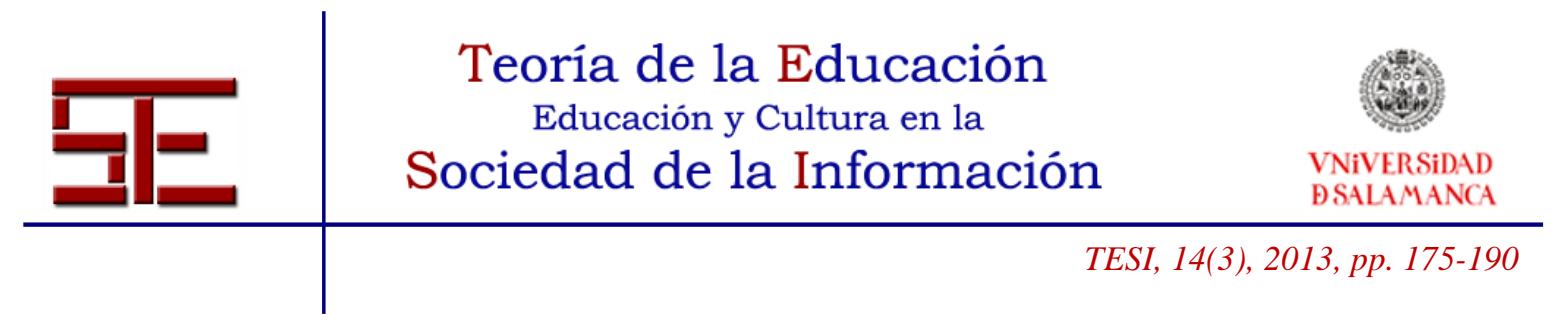

\title{
ENTORNOS HIPERTEXTUALES Y EDUCACIÓN
}

Fecha de recepción: 12/06/2013; fecha de aceptación: 05/07/2013; fecha de publicación: 30/11/2013

Javier Argos

argosj@unican.es

Universidad de Cantabria

Pilar Ezquerra

ezquermp@unican.es

Universidad de Cantabria

\begin{abstract}
“Quizás esperaba usted capas, marcos y los lentos despliegues del servidor incluidos o las superficies de páginas que recorren la pantalla sin alias. En lugar de eso, tenemos poesía y nudos autorreflexivos de hilos planteados difuminados como estelas de avión, ante la helada persistencia de vientos invisibles en los altos cielos cerúleos. Lo que haga usted con eso, será lo que literalmente haga, modelándolo por y para sí mismo, encontrándole significado de luz y de memoria, aun dándose cuenta de que su fundamentación y su duración no son más seguras que el aire alto y frio y los vientos fuertes y oscuros que soplan dentro de las órbitas exteriores de los electrones. Usted es el autor de lo que lea o escuche; es un proceso para el que quizá no se sienta adecuado, pero, una vez más, ¿quién lo está?”

(Michael Joyce, 2004, 145)
\end{abstract}

\section{EDUCACIÓN Y CONTEXTO TECNOLÓGICO}

En las últimas décadas estamos asistiendo a un verdadero aluvión de reflexiones teóricas y de estudios empíricos que abordan el sentido, lugar y papel que la educación debe y puede jugar, así como el formato que ella puede ostentar en el marco del actual escenario tecnológico. Todos esos trabajos afrontan ese importante reto epistemológico desde diferentes marcos paradigmáticos y desde enfoques metodológicos también diversos contemplando, unos y otros, propósitos variados y de diferente alcance y ámbitos de análisis que van desde los más macro-perspectivistas a los más específicos o micro-prácticos.

Ciertamente, la educación siempre ha tenido, dependiendo del contexto socio-histórico en el que se inscribiera, un protagonismo indudable, ubicándose en algún punto del continuo acotado por dos polos que, representando sus planteamientos más radicales, la conciben y proyectan bien desde su carácter claramente reproductor, bien desde aquel otro marcadamente transformador.

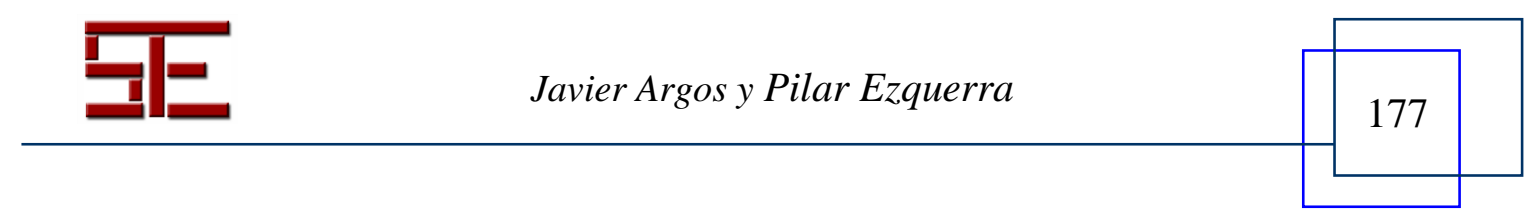




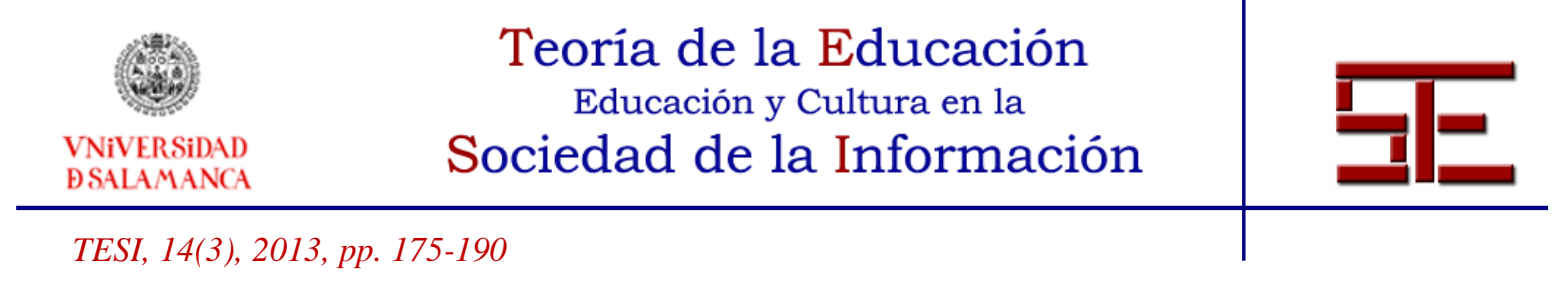

Si asumimos la afirmación de Bauman $(2007,12)$ de que "todas las sociedades son fábricas de significados" o, como él mismo se encarga de acentuar, son algo más que eso, llegando a concebirlas como "los semilleros de la vida con sentido", no inferiremos de forma errónea si entendemos algo que también es obvio para todos: el hecho de que el contexto social determina, afecta a lo educativo, influjo que, lejos de ser unidireccional, se lleva a cabo de forma dialógica.

Parece indiscutible que el contexto social afecta a lo educativo de múltiples modos. Así, cotidianamente, sobre el topos pedagógico se proyectan elementos tanto de expansividad como de encorsetamiento práxico. Las diferentes expectativas que cada sociedad se plantea en relación con la educación en general y con la escuela en particular tienen mucho que ver con el imaginario construido socio-históricamente en ese escenario concreto y que, en muchas ocasiones, no emerge de la necesaria reflexión sosegada y crítica sino, por desgracia, desde miradas superficiales, estereotipadas y prejuiciosas que arrastran a lo educativo a asumir demandas, si no exigencias, en manifiesta contradicción e incoherencia con el sentido y papel que debería de tener.

Una verdadera relación dialógica entre los contextos social y educativo pasaría por centrar más nuestra atención en los ámbitos semánticos, esto es, de significado, que en aquellos otros fundamentalmente ubicados en los elementos más superficiales o periféricos de ambos contextos o sistemas. Desde nuestra perspectiva, en esta necesaria relación profunda a acometer entre lo social y lo educativo deberían de jugar un importante papel lo que Assmann (2002) denomina interfaces y que entiende como zonas de comunicación o acción de un sistema sobre otro.

La construcción de interfaces entre los campos de sentido o de significado de las diferentes personas e instituciones integradas en los diferentes entornos vitales se convierte para nosotros en una terea fundamental si verdaderamente apostamos por generar un adecuado engranaje entre lo social y lo educativo.

Indiscutiblemente, uno de los principales elementos a engranar entre estos dos grandes ámbitos es el tecnológico. Para Buckinhan (2008) se ha producido un debate polarizado acerca del sentido y del papel que ha de jugar la tecnología en la educación. Así, mientras que algunos la promocionan exageradamente otorgándola un inmenso poder para potenciar e incluso liberar a los jóvenes, otros la ponen en tela de juicio entendiendo que es algo perjudicial o que, al menos, se trata de un sustituto no genuino de lo que podríamos concebir como verdadero aprendizaje. Ambas perspectivas se fundamentan en diferentes concepciones y supuestos específicos acerca de la infancia, del aprendizaje y del papel que la tecnología puede jugar en la sociedad en general. Como sostiene el referido autor,

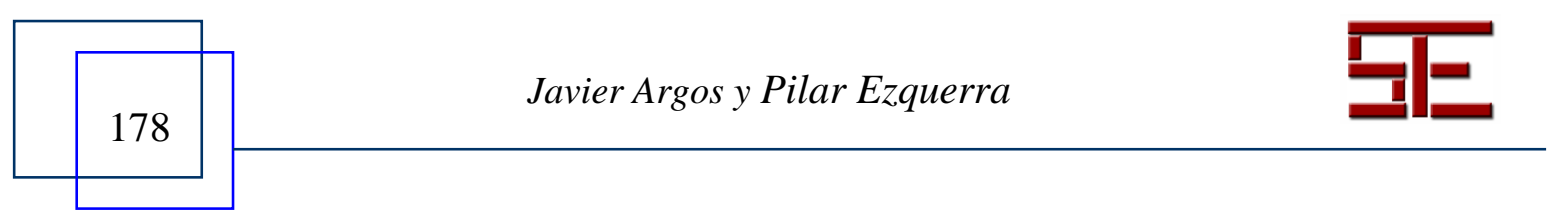




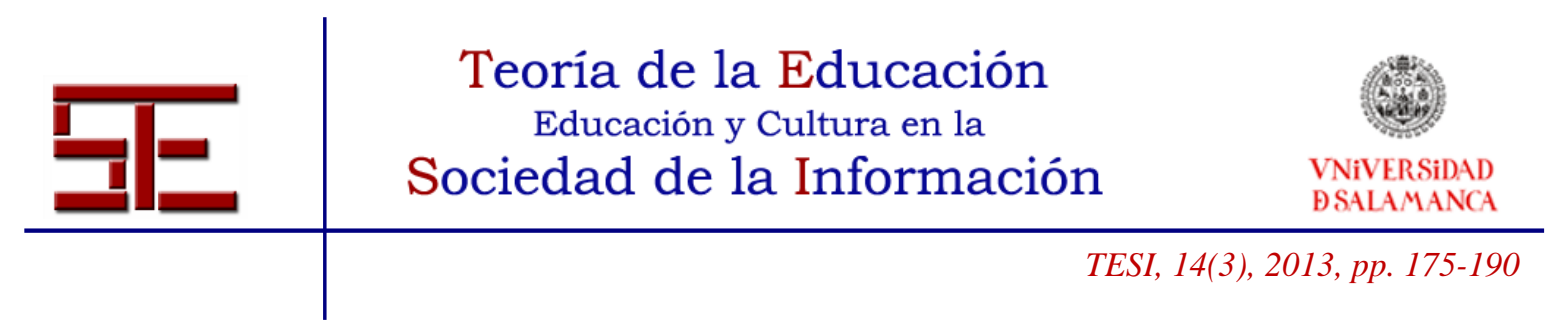

"En algunos sentidos, la polarización extrema de este debate puede tomarse como indicación de su inmadurez. Se condena con demasiada presteza a quienes cuestionan o rechazan el uso de la tecnología en la educación calificándolos de "tecnófobos" prehistóricos o "luditas", que oponen una resistencia irracional al "progreso", al tiempo que se define con demasiada facilidad a quienes predican los beneficios de la tecnología, cono inocentes o poco realistas en sus aspiraciones. Y mientras tanto, quedan sin respuesta preguntas fundamentales acerca de para qué podrían querer usar la tecnología los maestros y sus alumnos, y acerca de que deberíamos saber sobre la tecnología" (p.73).

Esta afirmación, a nuestro entender, pone en evidencia el asunto central al que deberíamos de atender todos los que nos dedicamos a la reflexión pedagógica, esto es, el de las preguntas fundamentales que otorgan sentido al hecho de operar pedagógicamente de una u otra manera. Ella hunde sus raíces en el sentido, el valor y el lugar que las herramientas tecnológicas pueden y/o deben tener y jugar en el escenario educativo.

En el presente trabajo pretendemos analizar, de forma sintética, el marco desde el que la educación formal y sus principales actores (estudiantes y docentes) deberían de contemplar los entornos hipertextuales o, si se quiere, la hipertextualidad, entendiéndola no sólo como una herramienta específica de comunicación y de aprendizaje sino, también, como algo que se inscribe en el marco de los procesos mentales-cognitivos y, aún de forma más amplia, en los contextos actitudinal y filosófico de cualquier proceso pedagógico.

\section{LA COMPLEJA DIALÉCTICA ENTRE LOS MODELOS Y LAS HERRAMIENTAS EN EDUCACIÓN}

El profesor francés Philippe Meirieu constataba hace ya más de una década el peligro que podríamos padecer los educadores al sentirnos seducidos o, como él decía "fascinados", por ciertas herramientas que incorporamos a nuestra tarea pedagógica sin caer en la cuenta de que cualquier herramienta ha de inscribirse dentro de un determinado modelo, de una representación de la educación y de la pedagogía que se conforma y articula en torno a tres elementos: "una toma de posición sobre las finalidades, la movilización de conocimientos de carácter psicológico relacionados con el "funcionamiento" del sujeto que aprende y, finalmente, las modalidades de acción admisibles" (2001, 109-110). A estos tres elementos él los denomina, respectivamente, el polo de las finalidades, el polo de apuntalamiento psicológico y el polo praxeológico en el que, en sentido estricto, se ubicarían las herramientas.

Por desgracia, en muchas ocasiones, los educadores pensamos y desarrollamos los procesos pedagógicos desde miradas cortoplacistas, estrechas y apresuradas obviando, bien por ignorancia, bien por desconsideración, aquél otro planteamiento macro o contextualista que ha de presidir cualquier propuesta educativa que se precie

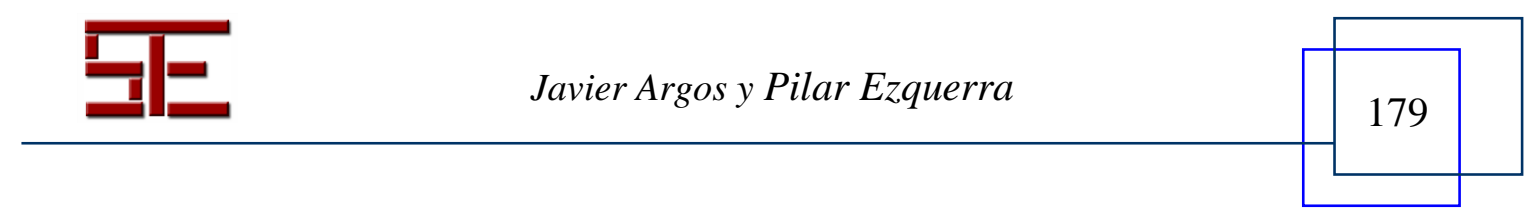




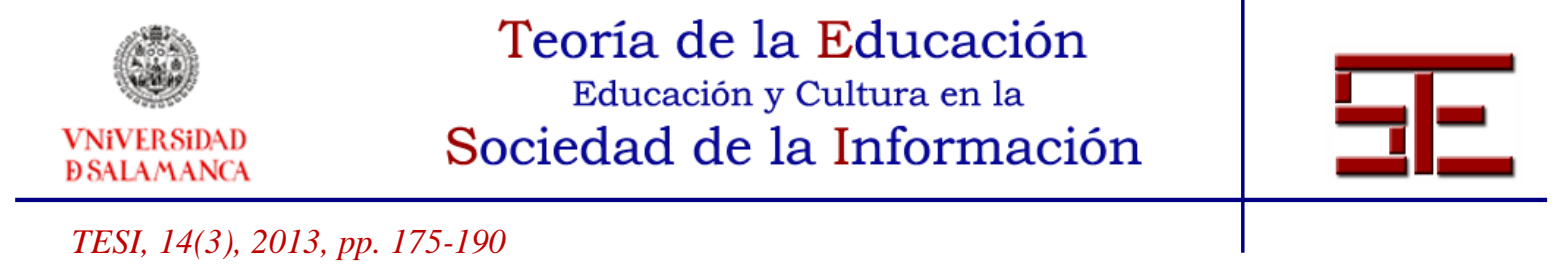

verdaderamente de serlo. Y cuando ello ocurre, esos procesos se descarnan de sus vectores más relevantes y potentes, fundamentalmente de aquellos que tienen que ver con el sentido de nuestra acción pedagógica, con los referentes de valor que la preñan y con la concepción antropológica que sostengamos en relación con las personas en ella implicadas.

Pero, aun siendo este hecho grave, más lo es si cabe el observar que, en muchas ocasiones, a los docentes ni siquiera se les permite abordar esa reflexión profunda sobre el sentido de su práctica profesional. Así, se les sitúa un tanto al margen de las importantes decisiones que conlleva la incorporación de las "herramientas" tecnológicas en el ámbito educativo. Aunque parece evidente que "los docentes necesitan entender los principios pedagógicos básicos que gobiernan la aplicación de la tecnología en los procesos de enseñanza y aprendizaje" (Satish, 2012, 53), no es menos cierto que "...el debate relativo a por qué, para qué, qué tecnologías incorporar, cómo hacerlo y en qué dirección le ha sido usurpado al profesorado, a quien se le ha colocado en situación de usuario y receptor de una toma de decisiones de expertos y técnicos que han decidido por ellos" (Rodríguez 2012, 135-136).

Nos encontramos muchas veces ante el sinsentido pedagógico de equiparar, situando en el mismo plano, el ámbito de las finalidades educativas y los de las metodologías y los recursos, otorgándoles similares potencialidades educativas y haciendo descansar sobre ellos el mismo grado de responsabilidad en el marco de los procesos educativos en los que estamos inmersos. Como apuntan García del Dujo y Martín (2002), debemos de incorporar la perspectiva pedagógica a la hora de analizar el proceso de inserción de la tecnología en el ámbito educativo, entendiéndola como un conjunto de instrumentos mediadores en el aprendizaje.

Desde nuestra perspectiva, la constatación de esta evidencia ha de llevarnos inexcusablemente a repensar los vectores que otorgan verdadero sentido a la práctica pedagógica, considerando los relacionados con los métodos y los recursos pedagógicos como subordinados a aquellos otros de carácter teleológico y axiológico que deberían de ser los axiales y, por tanto, con hegemonía referencial. Indudablemente, unos y otros, orientados en la misma dirección, han de aportar sinergias generadoras de esa anhelada coherencia pedagógica que se convierte en axioma, a la vez que en reto, de toda práctica pedagógica que sea valiosa y comprometida.

Por desgracia, en ciertas prácticas educativas, no sólo está ausente dicha coherencia sino que, con frecuencia, se constatan en ella disonancias funcionales entre los ámbitos o elementos anteriormente referidos. Así, por lo que respecta al ámbito de los recursos pedagógicos, las relaciones que en ocasiones éstos mantienen con las finalidades explícita o latentemente contempladas, lejos de ser de subordinación se perciben como de complementariedad -si no de hegemonía respecto de ellas- y, cuando esto ocurre,

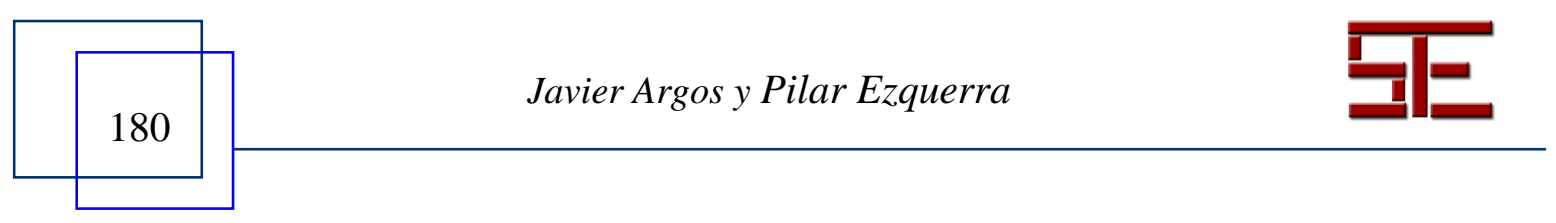




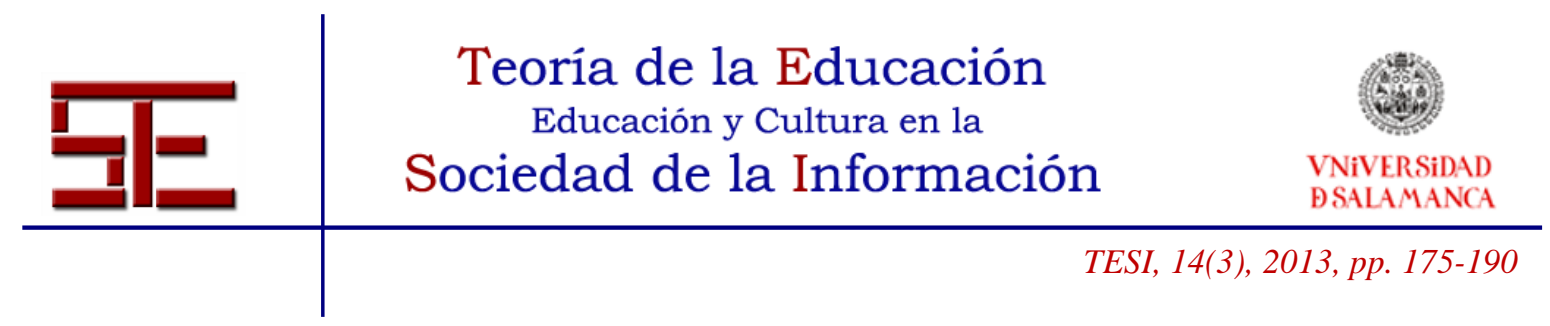

estamos invirtiendo el sentido de la reflexión pedagógica y, en consecuencia, el buen hacer de la práctica educativa que se genera desde ella.

Centrándonos en el ámbito de la tecnología educativa y, en concreto, del estudio de los contextos de innovación y de buenas prácticas que se llevan a cabo con las TIC en el entorno educativo, parece desprenderse la idea de que en ellos juegan un papel fundamental los objetivos educativos y la interpretación del valor pedagógico otorgado a las TIC (Colás y Casanueva, 2010). A partir de la revisión de los estudios sobre la integración de las TIC en el sistema educativo que estos investigadores realizan se constata que dichas tecnologías pueden contemplarse como elementos facilitadores de la consecución de diferentes tipos de propósitos. En este sentido, apuntan lo siguiente:

“Así en algunos centros toman la opción de centrar su esfuerzo en la provisión de las TIC, entendiendo que éstas tienen consecuencias en el desarrollo de competencias tecnológicas en los estudiantes. En otros casos la interpretación pone el acento en el potencial de las TIC para que el alumno alcance autonomía en el aprendizaje" (pp. 125-126).

Como señalábamos al comienzo de nuestro trabajo, esta reflexión pretende focalizarse y poner el acento en los entornos hipertextuales en la medida en que el hipertexto se ha convertido en una herramienta emergente que preside nuestros contextos y redes digitales.

El hipertexto, esa "galaxia de significantes" (que no "estructura de significados") tal como la concibe Ronald Barthes (1980, cit. Calderoni y Pacheco, 1998) creemos que también debería de ser sometido a consideración pedagógica desde la perspectiva de "gran angular" anteriormente apuntada. Considerar al hipertexto, al igual que lo hace Gilster (1997), como un elemento importante en la "nueva geografía de la información", aunque conceptualmente no sea algo tan nuevo como pudiera parecer al encontrar sus antecedentes en las notas a pie de página de las obras impresas (Pujolà y Montmany $(2007,3)$ no debería de ser óbice para nuestra cautela a la hora de sopesar las bondades y las debilidades que puede comportar la incorporación de la hipertextualidad a la práctica pedagógica.

\section{COGNICIÓN E HIPERTEXTUALIDAD}

Plantearse en clave de reflexión teórica el sentido y valor de lo hipertextual en el escenario pedagógico nos lleva inexcusablemente a pensar, aunque sea de forma sucinta, sobre los procesos cognitivos que se encuentran con ella vinculados y, también, sobre el tipo de narrativas que se generan desde los entornos virtuales y el valor que ellas tienen en los procesos de construcción de conocimiento.

Como apuntan los profesores García Carrasco, Gargallo, García Manzano y Sánchez i Peris $(2012,19)$, "la lógica hipertextual genera estructuras argumentativas discontinuas y los significados no emergen de los objetos mismos, sino de sus conexiones en una

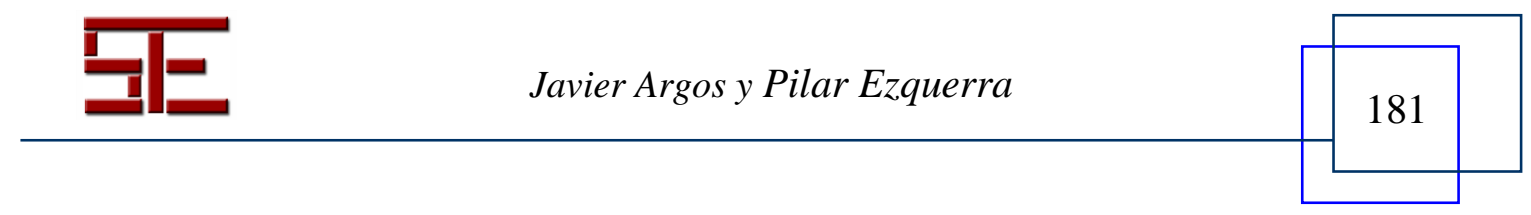




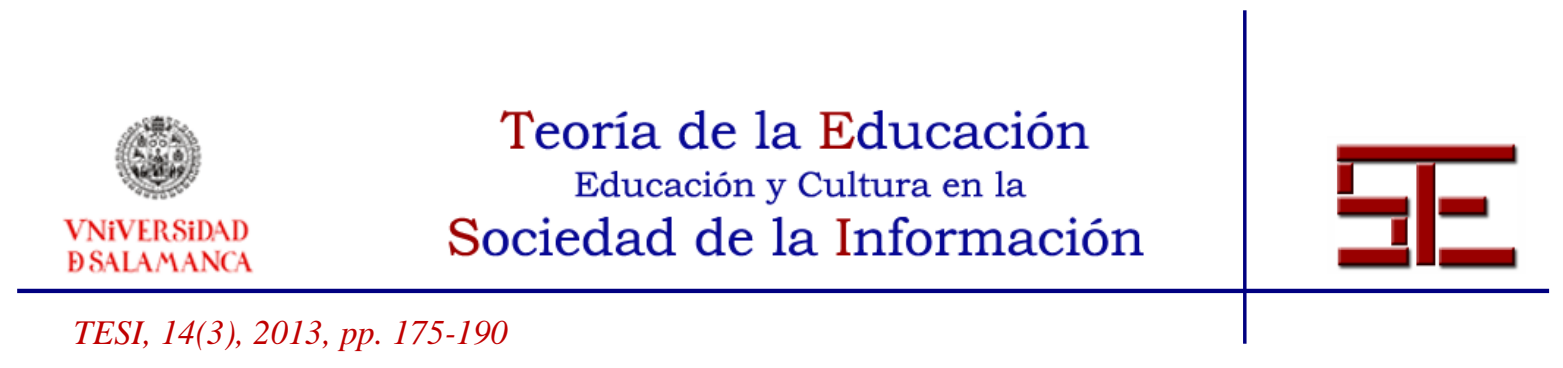

intrincada malla reticular" y se sustentaría en una nueva forma de pensamiento rizomático apoyado en la colaboración. Ese tipo de pensamiento, defendido entre otros, por Deleuze y Guattari, y que ha sido también fuente de críticas (Reynoso, 2012), estaría estrechamente vinculado con la Teoría de la Flexibilidad Cognitiva (Cognitive Flexibility Theory) auspiciada por Spiro y colaboradores. Esa flexibilidad cognitiva supondría la competencia para reestructurar de forma espontánea nuestro conocimiento para que pueda adaptarse de múltiples formas a las demandas de los cambios situacionales (Spiro \& Jehng, 1990). Este "nuevo constructivismo" como lo denominan Spiro et al. $(2007,8)$ "responde a las dificultades que presenta la adquisición de un conocimiento avanzado en dominios [marcos] mal estructurados" e integraría elementos relacionados con el aprendizaje, con la representación mental y con la instrucción.

En los sistemas hipertextuales, el alumno puede desplazarse por la información en función de diferentes criterios como, por ejemplo, la relevancia que tenga ella para él, el grado de interés y de curiosidad que despierte, el nivel de experiencia que posea el alumno, la necesidad de información que éste tenga o los motivos que hayan generado la demanda de información (Gros et al., 1997). Para estas profesoras, estos sistemas son los más adecuados para llevar a cabo aprendizajes complejos o avanzados aunque no lo serían en contextos de aprendizaje de carácter básico o introductorio.

Aunque, desde las anteriores argumentaciones pudiera desprenderse la idea de que lo hipertextual se conforma como un poderoso marco de pensamiento y de aprendizaje no es menos cierto, como nos apuntan Asensio, Álvarez y Rodríguez $(2012,22)$ que “...se ha abierto ya en los últimos años un cierto debate por lo general bastante polarizado entre quienes consideran que las generaciones que han crecido en el mundo de las pantallas están desarrollando magníficos cerebros hipertextuales y quienes consideran que esos mismos cerebros, con el tiempo, serán incapaces de articular un pensamiento que precise varias oraciones para ser expresado".

En un reciente artículo de prensa de José Antonio Marina (2013) en el que se hace referencia de forma muy sintética a nuestros procesos de memoria y de creatividad, el profesor sostiene que “...la memoria creadora se basa en ricas redes de información, tupidamente conectada, y en la agilidad para recorrerlas". Para él, los campos semánticos están cruzados por una red de caminos mentales y Google no hace otra cosa más que intentar copiar nuestras redes interiores. Ciertamente, puede ser un auxiliar fantástico, pero su aprovechamiento dependerá de nosotros mismos.

En la misma línea se posicionan Bera \& Liu (2006) cuando afirman que “...aunque las capacidades hipertextuales de los entornos hipermedia teóricamente imitan el modo en el que se estructura el conocimiento en la memoria humana no desempeñan, por sí mismos, una función cognitiva" (p. 296). Los resultados de la investigación llevada a cabo por estos investigadores estadounidenses apuntan a la conveniencia de trabajar desde enfoques contextuales que, conformados por variables de diferente tipo, generen

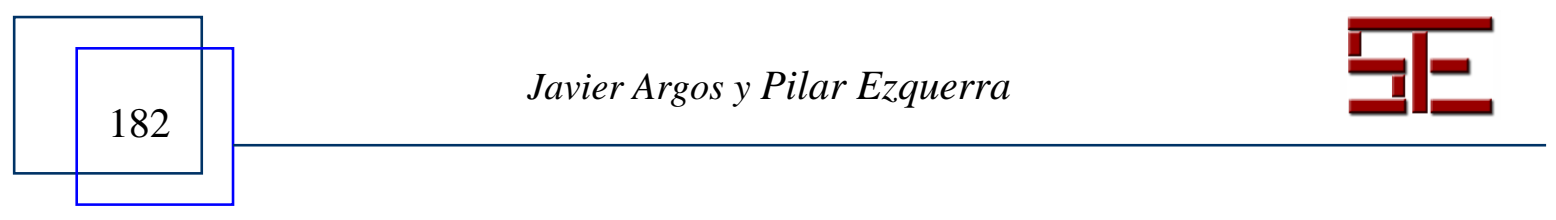




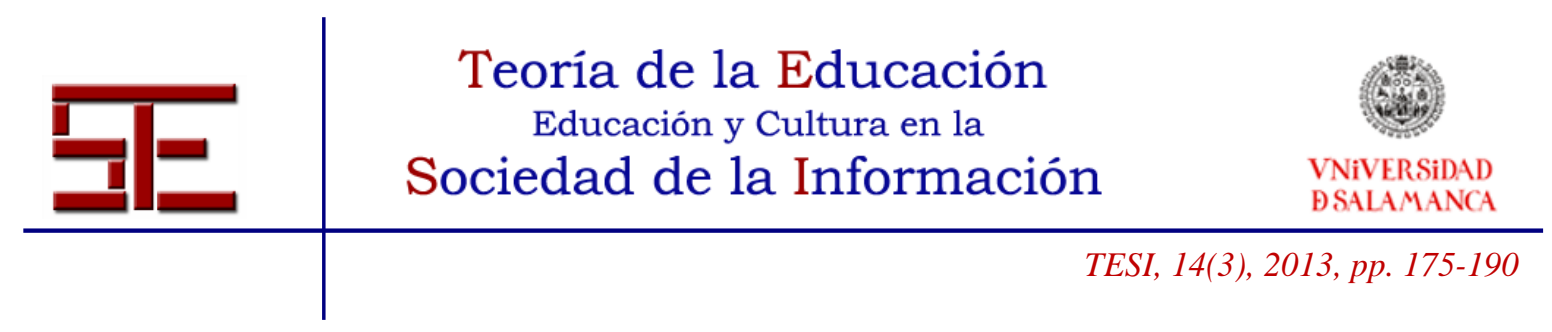

condiciones óptimas de aprendizaje. Para ellos, el papel del estudiante no debería de limitarse a navegar a través de los hipermedia sino que, más bien, sería el de utilizar una serie de herramientas para explorar el entorno, obtener información, probar hipótesis y generar soluciones.

Una de las derivadas que emergen con fuerza cuando analizamos esta problemática desde enfoques contextuales es la de las diferencias individuales de los alumnos, esto es, aquella generada por los múltiples contextos personales con los que cotidianamente convivimos en el desarrollo de la práctica pedagógica. Una buena muestra de ello lo constatan Chen, Fan \& Macredie (2006) en la revisión que efectúan acerca del valor de los sistemas hipermedia en función del grado de experiencia y de dominio que posean los alumnos en relación con el ámbito de los aprendizajes a acometer. Así, ellos constatan que los aprendices "expertos" se desenvuelven mejor que los noveles en los sistemas de aprendizaje hipermedia, fundamentalmente debido a su más profundo conocimiento de la materia, y que es el que les permite poder conducir mejor su exploración en dichos entornos. En este mismo sentido se manifiesta Granchetti (2011) cuando afirma que "si bien un hipertexto puede mostrar una compatibilidad maravillosa con el constructivismo, también es cierto que presenta mayores riesgos de naufragio cognitivo para los más principiantes".

Pero, al igual que sugieren Chen, Fan \& Macredie (2006) en las conclusiones de su estudio, también han de ser tenidas en cuenta otra serie de variables como, por ejemplo, las diferencias vinculadas al género, a los antecedentes culturales o a los estilos cognitivos de los alumnos.

Ciertamente, el efectuar un estudio riguroso acerca del valor pedagógico de los entornos hipermedia entraña una gran dificultad. Así, en el interesante trabajo de Scheiter \& Gerjects (2007), junto con la constatación de las potenciales bondades que ostenta para el estudiante este tipo de entornos y, más concretamente, el elevado control que él puede tener sobre su propio proceso de aprendizaje, se presentan una serie de potenciales dificultades que podemos encontrar y que están relacionadas con una serie de variables.

Así, entre los argumentos favorables que los sistemas multimedia podrían tener en el aprendizaje respecto de aquellos otros entornos en el que éste se lleva a cabo de una forma más controlada, estos investigadores germanos apuntan los siguientes: 1) Las estructuras hipermedia son el reflejo de la mente; 2) Incrementan el interés y la motivación; 3) Se adaptan a las preferencias y a las necesidades cognitivas; 4) Tienen potencialidad de cara a un proceso de recabar información activo y constructivo y, 5) Permiten la adquisición de habilidades autorregulatorias.

Pero, como contrapartida, en el referido trabajo también se ponen de manifiesto tres tipos de variables que pueden afectar negativamente al aprendizaje de los alumnos. En

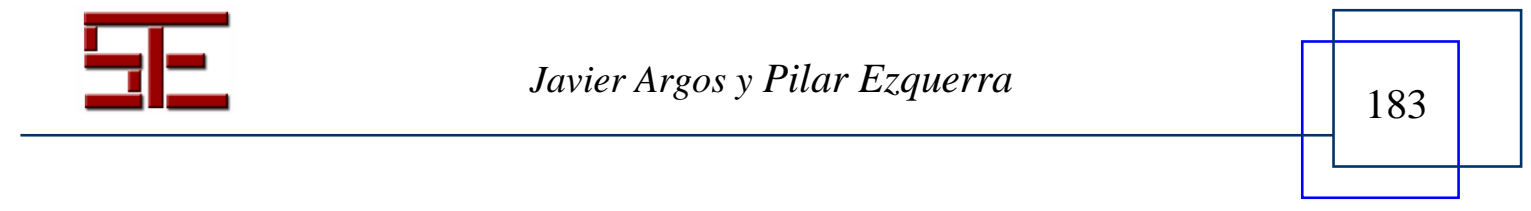




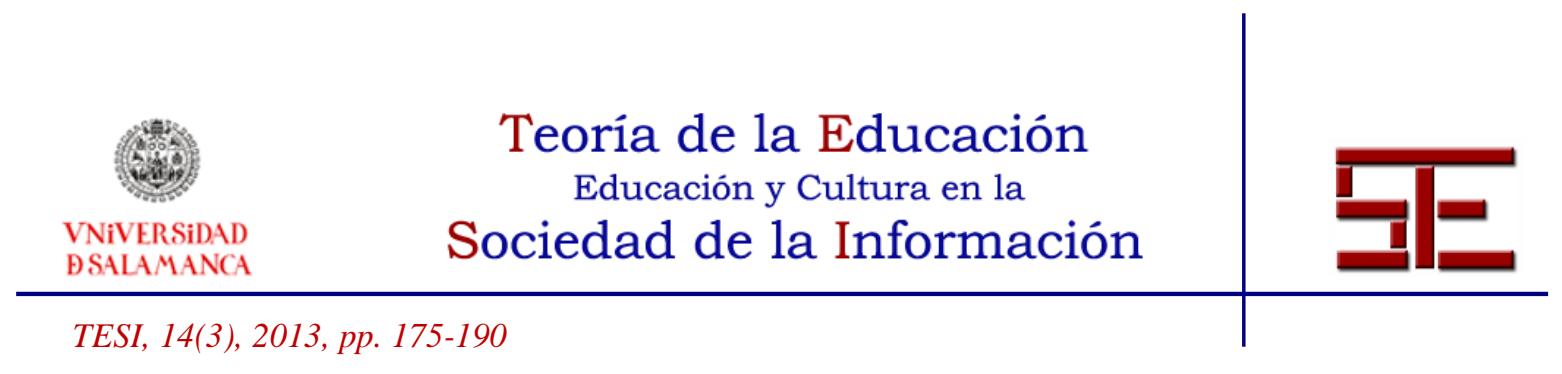

concreto, son los problemas relativos a: 1) Desorientación; 2) Distracción y, 3) Sobrecarga cognitiva.

En concreto, los problemas de desorientación se producen cuando los usuarios (en nuestro caso, podríamos referirnos a los alumnos) ignoran el lugar de la red en el que se encuentran, así como el lugar de ésta hacia el que desean ir y el modo de hacerlo. Para estos investigadores, parafraseando lo apuntado por Foss (Foss,1989, en Scheiter \& Gerjects, 2007, 290), "la desorientación es inherente al hipermedia debido a que éste es el resultado directo de amplias y complejas estructuras de datos donde sólo se puede mostrar simultáneamente una pequeña parte de la información posible" (290) aunque investigaciones posteriores han constatado soluciones tecnológicas a este problema vinculadas, fundamentalmente, al desarrollo de diferentes herramientas de navegación y de redes estructuradas.

Por su parte, los problemas de distracción suelen aparecer cuando los aprendices que se encuentran en un entorno hipermedia navegan y acceden a la información guiados por su interés que puede ir variando al sentirse condicionado por el marco o contexto que, en cada momento, proporcione ese entorno.

Por último, la denominada sobrecarga cognitiva también puede aparecer como un problema en el aprendizaje en este tipo de entornos, como consecuencia del alto grado de libertad otorgada al usuario a la hora de regular o controlar la navegación y, por ende, su curso de aprendizaje. Así, en el trabajo de Niederhauser et al. (2000) se constata que todas las decisiones que aquél ha de adoptar en el marco de su elevado grado de autonomía, genera en él una carga cognitiva adicional a su aprendizaje.

Como se puede apreciar del anterior análisis, los entornos hipermedia son generadores de claroscuros en los que conviven dialécticamente potencialidades y debilidades que le son intrínsecas. De hecho, la revisión de la investigación efectuada por Scheiter \& Gerjects (2007) apunta y estudia diferentes tipos de razones a las que puede deberse la ambigüedad de los resultados por ella generados cuando ha tratado de valorar la efectividad de ese tipo de entornos de cara al aprendizaje.

\section{LA NECESARIA INCORPORACIÓN DE LO HIPERTEXTUAL A LA TOTALIDAD DEL ESCENARIO PEDAGÓGICO}

En este último epígrafe pretendemos adentrarnos sucintamente en una reflexión sobre la hipertextualidad que trascienda al mero plano praxeológico (de la herramienta) e, incluso, al psicológico (de las teorías sobre la cognición humana), para ubicarnos en otro más amplio, el educativo, que ejerce de sustrato profundo a la vez que de elemento delimitador de los escenarios potencialmente generadores de buenas prácticas pedagógicas.

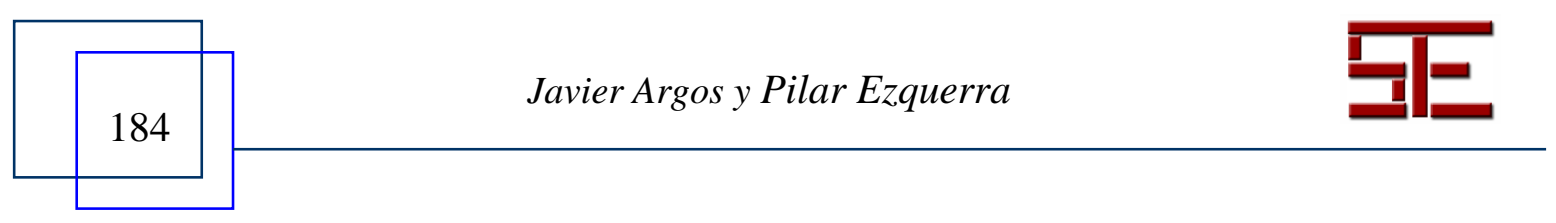




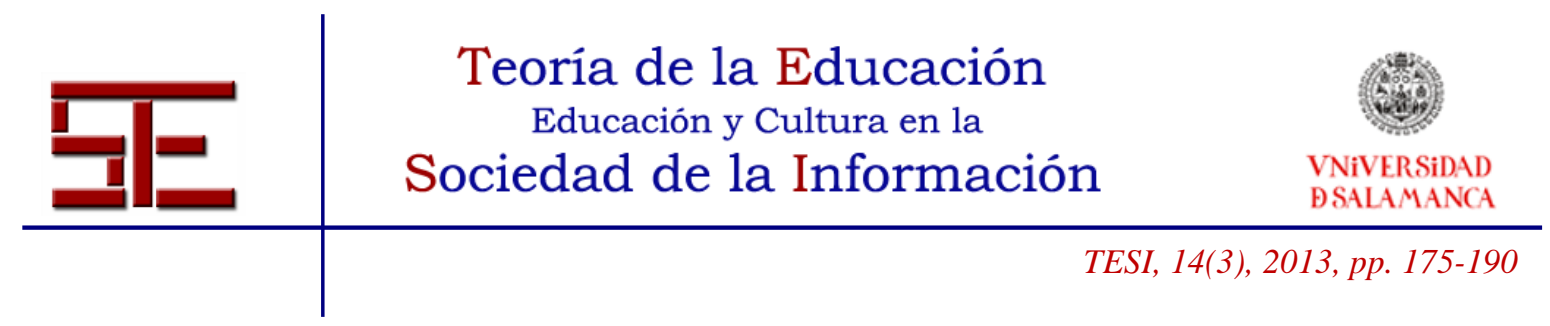

Como hemos ido constatando a lo largo del trabajo, la vigencia, la potencialidad educativa e, incluso, la controversia que generan los entornos multimedia es algo evidente. Ahora bien, si se observa, muchos de los análisis y argumentos de defensores y detractores se ubican en el plano del aprendizaje y, por extensión, del aprendiz (en nuestro caso, del alumno) como si la educación pudiera llevarse a cabo a espaldas de un proceso profundamente dialógico e interpersonal.

Ciertamente, puede argumentarse que, de algún modo, muchos sistemas y recursos hipermedia son lo suficientemente sofisticados como para entender que esa relación dialógica se produce aun cuando se lleve a cabo de un modo no necesariamente presencial. Pero, indudablemente, una relación pedagógica profunda y verdadera reclama la presencia, la visibilidad del educador. Ese que "está ahí", cercano, "andamiando" los aprendizajes, acompañando y guiando al alumno como el sentido etimológico del término pedagogo pretendía connotar.

Es por ello por lo que creemos que, la hipertextualidad como herramienta educativa no debería de ubicarse sólo en el ámbito del aprendizaje sino que, inexcusablemente, nos reclama también su presencia en el de la enseñanza contemplándose, dentro de ésta, tanto en el plano personal (de cada docente) como en el institucional-escolar. Postulamos que sólo desde esta consistencia de planos y de ámbitos del escenario pedagógico será desde donde esa herramienta pueda encarnarse y proyectarse en todos los planos anteriormente aludidos: el "técnico", el "cognitivo" y el "educativo".

Responder seriamente a esta demanda de consistencia en un aprendizaje multimedia como éste, nos debería de llevar a entenderlo desde la metáfora de la "construcción del conocimiento", en la que el alumno es un activo constructor de significados, el docente un guía cognitivo y el multimedia un facilitador de un sistema de guía cognitivo. Supondría ubicarse en un enfoque de enseñanza multimedia centrado en el alumno más que en aquel otro que se centra en la tecnología (Mayer, 2005).

Por lo tanto, reiterando lo dicho, plantearnos el sentido, valor y lugar que podría tener lo hipertextual en el ámbito de la educación formal supondría considerarlo no sólo desde la perspectiva del alumnado sino, también, hacerlo isomórficamente desde la que podríamos denominar hipertextualidad docente.

A nuestro entender, esa hipertextualidad docente se construye desde diferentes vectores o vertientes que la integran y que le otorgan (o le hacen adolecer de) consistencia interna. En concreto, incorporar lo hipertextual a la práctica pedagógica supone para el docente contemplarlo desde las vertientes epistemológica, metodológica y actitudinal.

Las implicaciones que para el docente tendría el analizar la cuestión desde la vertiente epistemológica estarían vinculadas con la asunción por su parte de que los sistemas de conocimiento no son lineales sino fundamentalmente rizomáticos y complejos y, también, el entender desde planteamientos de carácter holístico, que las configuraciones

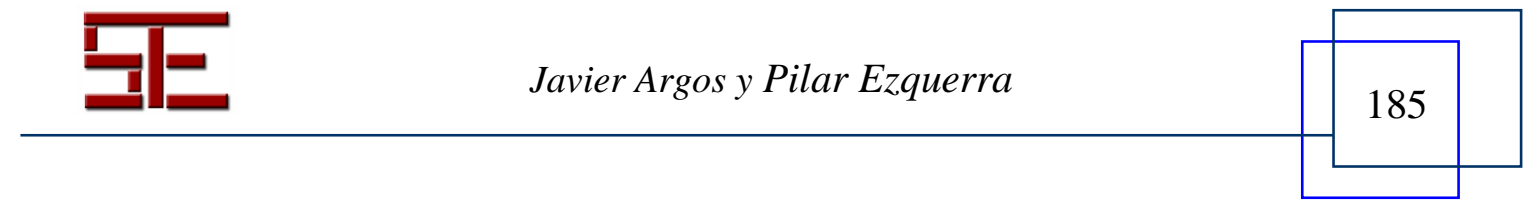




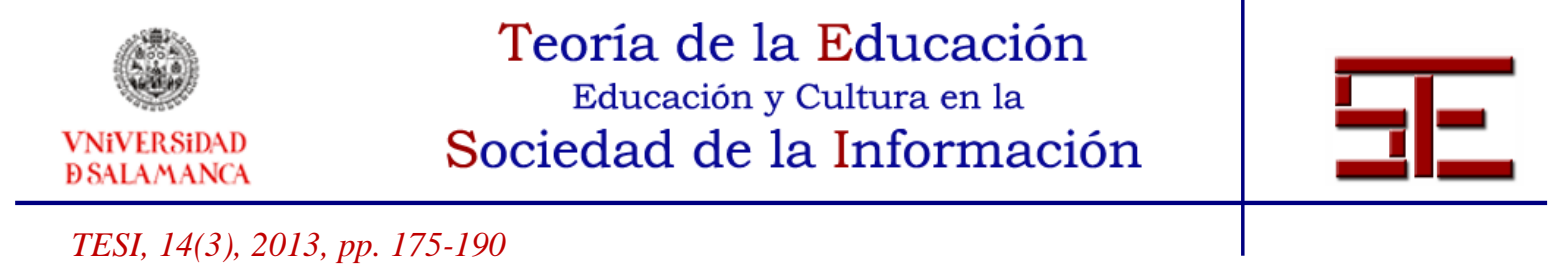

o totalidades de elementos constituyen importantes referentes para la construcción de significados coherentes y sólidos por parte de los alumnos.

En cierto modo, esta concepción de carácter epistémico es determinante y ocupa un importante lugar en el pensamiento de los docentes y en la forma de concebir su práctica pedagógica. Además, sus formas de pensar, sus percepciones y concepciones “...aunque no constituyen por sí solas la cultura de un centro [...] se consideran como uno de los principales pilares de la institución educativa" (De Pablos, Colás y Villaciervos, 2010, 193).

Las concepciones epistemológicas de los docentes cobran aún más protagonismo y se convierten en importantes referentes, cuando se llevan a cabo propuestas sustentadas en el trabajo colaborativo (Giraldo, 2008). De hecho la investigación desplegada por esta investigadora en el ámbito de la educación superior deja constancia de las reservas epistemológicas que pueden aparecer entre las diferentes disciplinas y sus correspondientes docentes, en el marco de lo que ella denomina horizonte interdisciplinario.

La relevancia de la vertiente epistemológica también la constata Carlos Monereo (2005) cuando en uno de los puntos de su decálogo que concreta las orientaciones de lo que él denomina una práctica edumática, aboga por la importancia que ha de tener la coherencia del docente con su modelo epistemológico. Como él apunta, cualquier decisión de utilizar las TIC en el ámbito escolar debería de estar enmarcada en una determinada opción acerca de lo que significa aprender y, consiguientemente, enseñar.

Por su parte, considerar lo hipertextual desde la vertiente metodológica supondría tener muy presente que las interacciones que ese nuevo marco puede generar en el alumno son de diferente índole (interacciones con el contenido, con el profesor, con los compañeros y con el alumno mismo) (Barberá, 2004, 127-136). Para esta profesora, el docente ha de apoyar a los alumnos haciendo que éstos se sientan que están aprendiendo en un entorno cómodo con una guía sólida, debiendo de estar liderada su tarea por la empatía didáctica y no limitarse a responder a los problemas que le puedan ir surgiendo al alumno. En este contexto, el docente deber evitar tres grandes peligros: el del retorno al enfoque transmisivo (en lugar de desarrollar su práctica desde un enfoque constructivo y colaborativo); el de la tensión entre la individualización y la "estandarización flexible" $\mathrm{y}$, por último, el de la posible sensación de pérdida de autoridad.

Asimismo, infiriendo de lo apuntado por Burbules (2004), supondría no limitarnos como docentes a que el alumno se relacione de forma binaria y estática con los hiperenlaces sino que, tendríamos que tratar de conseguir que ese entorno hipertextual generase actividades de segundo orden que lleven al alumno a cuestionar y a interrogar críticamente los enlaces y a resistirse a aquellas asociaciones que invitan a establecerse

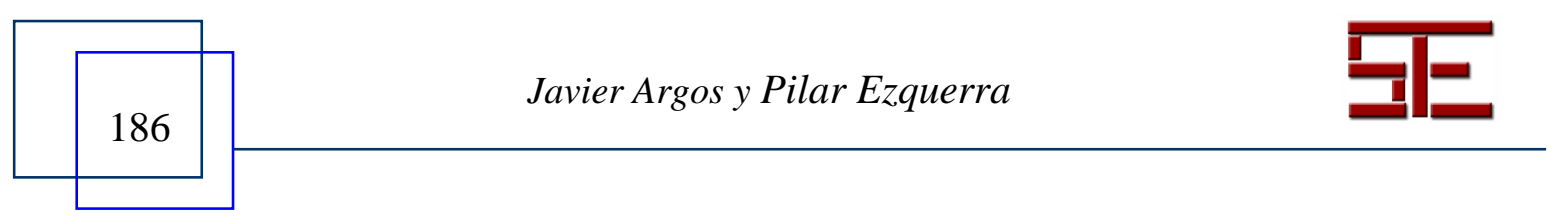




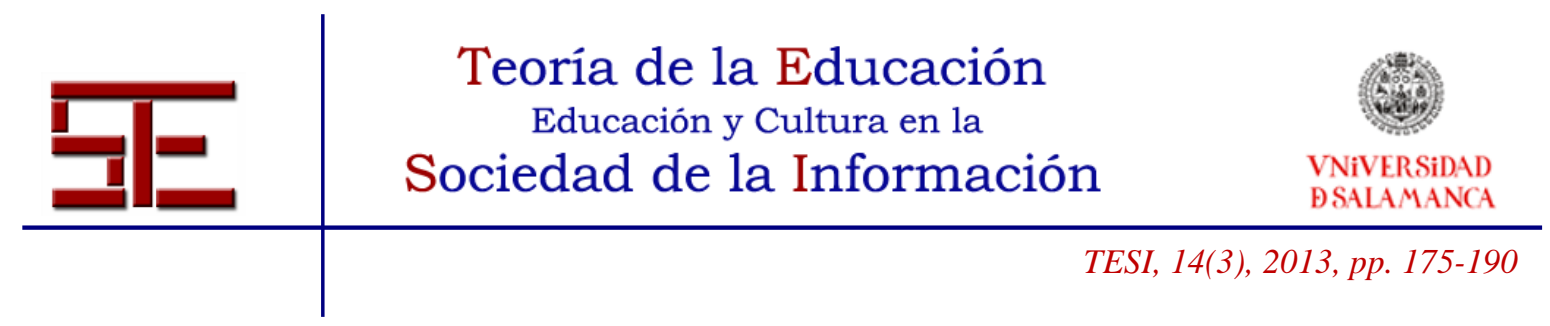

sin pensar. Por último, implicaría reflexionar acerca de las posibilidades y limitaciones que comporta este tipo de aprendizajes sustentados en los hipermedia: tipos de aprendizajes y/o de tareas más adecuadas, consideración de las diferencias individuales en este tipo de aprendizajes, conveniencia de que el docente proporcione organizadores previos que ayuden a percibir la navegación y la percepción de la estuctura... (Dillon \& Jobst, 2005).

En definitiva, como apunta el ya referido Granchetti (2011), el rol de los docentes en cuanto que facilitadores del aprendizaje de sus alumnos debería de ser dinámico y versátil, de tal forma que en el proceso inicial de hiperlectura su rol fuera de guía para, paulatinamente, irse éste desvaneciendo conforme el aprendiz va adquiriendo los criterios de navegación y construyendo las conexiones hipertextuales. Algo que, como el autor reconoce, no es fácil de lograr.

Finalmente, considerar la hipertextualidad docente desde la vertiente actitudinal supone atender a una dimensión que, aun habiendo sido frecuentemente desconsiderada o desatendida en el ámbito pedagógico desde planteamientos y filosofías educativas de corte tecnocrático y eficientista, resulta determinante en cuanto que se ubica en el sustrato en donde se alojan los vectores que dan sentido a nuestra práctica.

Supondría enfatizar dos de las cuatro dimensiones formativas que contemplan Area, Gros y Marzal (2008) en el marco del modelo educativo integral para la alfabetización en el uso de las TIC, aplicándolas en este caso al ámbito específico del docente: la actitudinal y la axiológica y que, a nuestro entender, van de la mano.

Así, desde la dimensión actitudinal, el docente debería de desarrollar actitudes racionales ante la tecnología (ni tecnofóbicas ni tecnofílicas) así como actitudes positivas en la comunicación y, desde la axiológica, tomar conciencia de la no neutralidad de las TIC, asumiendo aquellos valores y criterios éticos necesarios para el adecuado uso de la información y de la tecnología.

Nuestro reto no sólo sería atender a esta dimensión actitudinal sino, además, velar porque se produzca una adecuada articulación entre ella y las otras dos dimensiones o vertientes. Así, la hipertextualidad debería de engranarse entre todas ellas, entendiéndose no sólo como una herramienta periférica -en ocasiones, hasta nuclearde nuestra tarea pedagógica sino, también y fundamentalmente, como algo más amplio y genérico que cobra sentido y realidad en nuestras aulas debido al valor que le otorga el docente en cuanto que constructo potente para entender y hacer entender la realidad compleja y reticular en la que estamos inmersos.

En definitiva, la reflexión que se ha efectuado en el presente trabajo servirá de poco si, como a veces ocurre, las miradas o enfoques que se efectúan y las prácticas que se generan desde el binomio educación-tecnología, son superficiales y/o reduccionistas. Porque, como nos apunta Buckingham (2008, 231), “en última instancia, es necesario

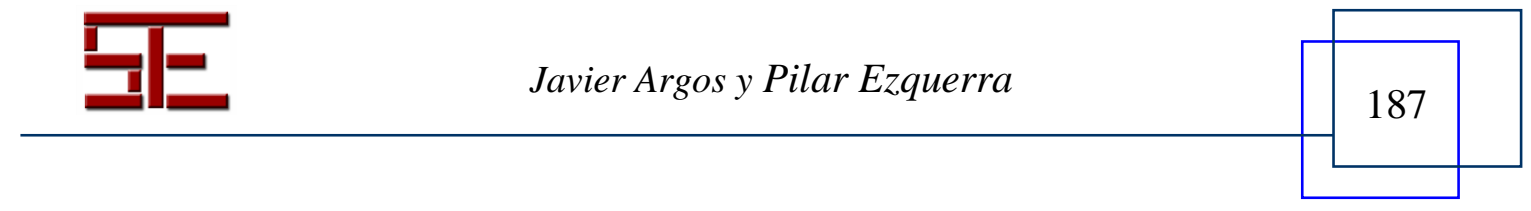




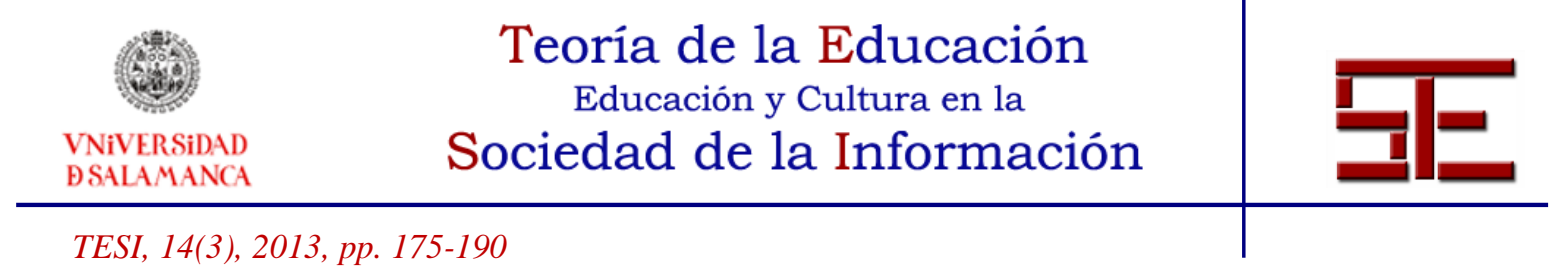

que dejemos de pensar en términos de tecnología y empecemos a pensar sobre un nuevo enfoque acerca del aprendizaje, la comunicación y la cultura".

\section{REFERENCIAS}

Area, M., Gros, B. y Marzal, M.A. (2008). Alfabetizaciones y tecnologías de la información y la comunicación. Madrid: Síntesis.

Asensio, J.M., Álvarez, I., Vega, F. y Rodríguez Neira, T. (2012). Las competencias de los profesionales de la educación hoy. La transformación de la práctica educativa. En L. García Aretio (Ed.), Sociedad del Conocimiento y Educación (pp. 193-213). Madrid: Uned.

Assmann, H. (2002). Placer y ternura en la educación. Hacia una sociedad aprendiente. Madrid: Narcea.

Barberá, E. (2004). La educación en la red. Actividades virtuales de enseñanza y aprendizaje. Barcelona: Paidós.

Bauman, Z. (2007). La sociedad individualizada (2 ed.). Madrid: Cátedra.

Bera, S. \& Liu, M. (2006). Cognitive tools, individual differences, and group processing as mediating factors in a hipermedia environment. Computers in Human Behavior, 22, 295-319.

Buckingham, D. (2008). Más allá de la tecnología. Aprendizaje infantil en la era de la cultura digital. Buenos Aires: Manantial.

Burbules, N.C. (2004). La red como un lugar retórico. En I. Snyder (Comp.), Alfabestismos digitales. Comunicación, Innovación y Educación en la Era Electrónica (pp. 115-128). Málaga: Aljibe.

Calderoni, J. y Pacheco, V. (1998). El hipertexto como nuevo recurso didáctico. Revista Latinoamericana de Estudios Educativos. 28 (3/4), 157-181.

Chen, S.Y., Fan, J. \& Macredie, R.D. (2006). Navigation in hypermedia learning systems: experts vs. novices. Computers in Human Behavior, 22, 251-266.

Colás, P. y Casanova, J. (2010). Variables docentes y de centro que generan buenas prácticas con TIC. Revista Teoría de la Educación: Educación y Cultura en la Sociedad de la Información.11(3), 121-147 [fecha de consulta 25/03/2013]. http://campus.usal.es/ revistas _trabajo/index.php/revistatesi/article/view/5791/5863

De Pablo, J. de, Colás, P. y Villaciervos, P. (2010). Políticas educativas, buenas prácticas con TIC en la comunidad autónoma andaluza" Revista Teoría de la Educación: Educación y Cultura en la Sociedad de la Información.11(1), 180-202

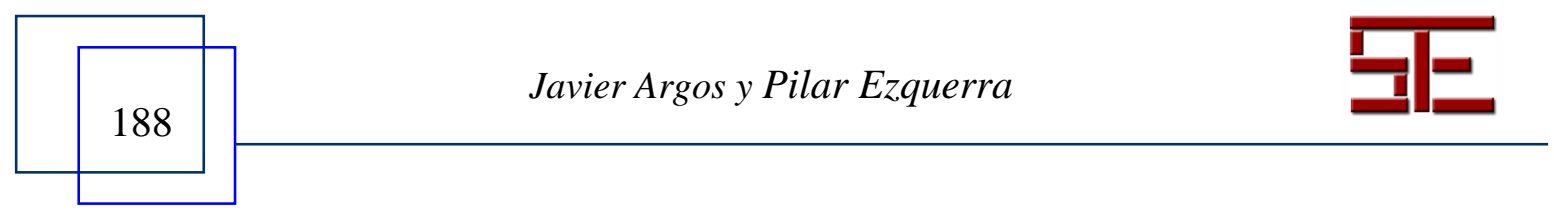




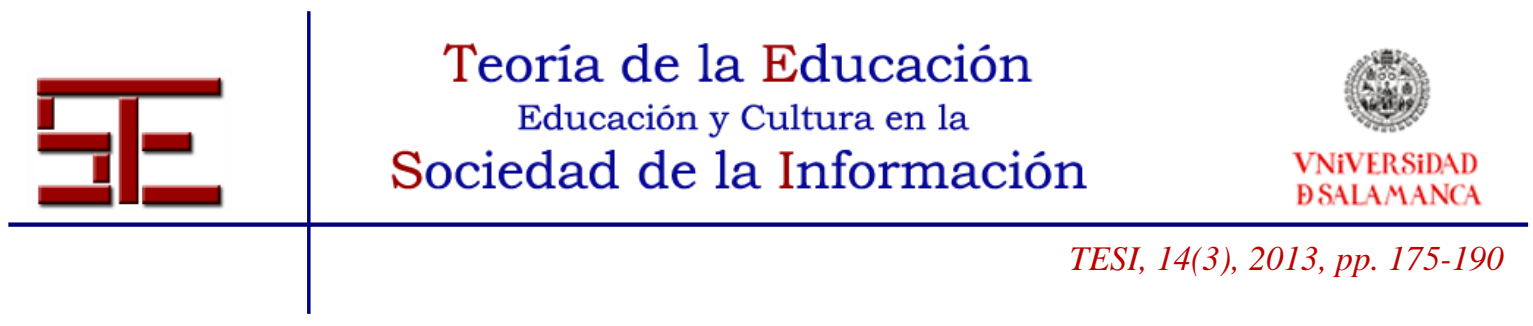

[fecha de consulta 25/03/2013]. http://campus.usal.es/ revistas _trabajo/index.php/revistatesi/article/view/5842/5868

Dillon, A. \& Jobst, J. (2005). Multimedia Learning with Hypermedia. En R.E. Mayer, (Ed.), The Cambridge Handbook of Multimedia Learning (pp. 569-588). New York: Cambridge University Press.

Foss, C.L. (1989). Detecting lost users: Empical Studies on browsing hipertext (Report No. 972, Programme 8).

García Carrasco, J., Gargallo López, B., García Manzano, A. y Sánchez i Peris, F. (2012). Nuevos modelos de aprendizaje en el contexto de la sociedad del conocimiento. En L. García Aretio (Ed.), Sociedad del Conocimiento y Educación (pp.305-339). Madrid: Uned.

García del Dujo, A. y Martín, A.V. (2002). Caracterización pedagógica de los entornos virtuales de aprendizaje. Teoría de la Educación. Revista Interuniversitaria, 14, 67-92.

Gilster, P. (1997). Digital Literacy. Canadá: John Wiley \& Sons Inc.

Giraldo, M.E. (2008). Metodología para la construcción colaborativa de hipertextos: el caso Hipernexus en educación superior. Revista Q. Educación, comunicación, tecnología, 3(5), 1-22. Extraído el 4 de marzo de 2013 de http://revistaq.upb.edu.co

Granchetti, H. (2011). La hipertextualidad del aprendizaje. Extraído el 1 de junio de 2012 de http://memoriastecno.blogspot.com.es/2011/11la-hipertextualidad-delaprendizaje.html

Gros, B. et al. (Coord.) (1997). Diseños y programas educativos. Pautas pedagógicas para la elaboración de software. Ariel: Barcelona.

Joyce, M. (2004).Una vez más: ¿quién lo es? Retórica posthipertextual. En I. Snyder (Comp.), Alfabetismos digitales. Comunicación, Innovación y Educación en la Era Electrónica (pp. 129-146.) Málaga: Aljibe.

Marina, J.A. (2013). Las Encinas. La Vanguardia, 12 de mayo de 2013. Extraído el 20 de mayo de 2013 de http://www.joseantoniomarina.net/articulo/las-encinas/

Mayer, R.E. (2005). Introduction to Multimedia Learning. En R.E. Mayer (Ed.), The Cambridge Handbook of Multimedia Learning (pp. 1-16). New York: Cambridge University Press.

Meirieu, Ph. (2001). La opción de educar. Ética y pedagogía. Barcelona: Octaedro.

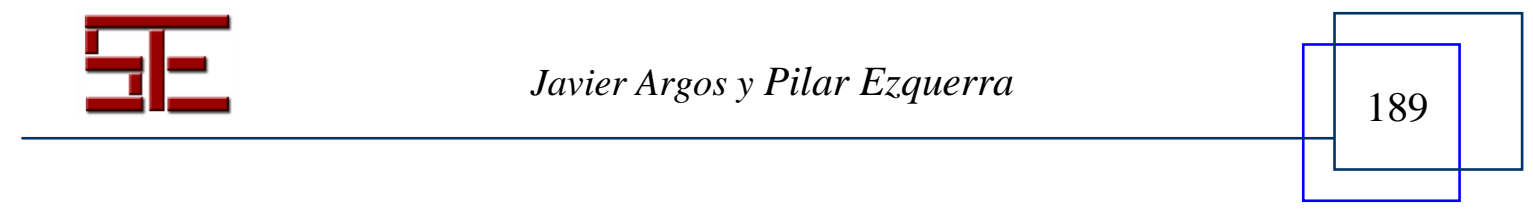




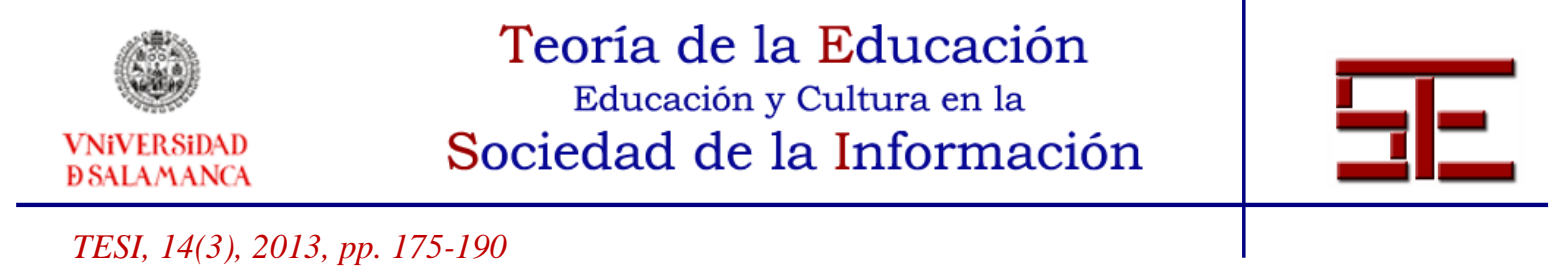

Monereo, C. (2005). Internet, un espacio idóneo para desarrollar las competencias básicas. En C. Monereo (Coord.), Internet y competencias básicas: aprender a colaborar, a comunicarse, a participar, a aprender (5-26).Barcelona: Graó.

Niederhauser, D.S. et al. (2000). The influence of cognitive load on learning from hypertex. Journal of Educational Computing Research, 23, 237-255.

Pujolà, J.T. y Montmany, B. (2007). Más allá de lo escrito: la hipertextualidad y la multimodalidad en los blogs como estrategias discursivas de la comunicación digital. Extraído el 19 de julio de 2012 de http://www.gabinetecomunicacionyeducacion.com/files/adjuntos

Reynoso, C. (2012). Árboles y redes: Crítica al pensamiento rizomático. Versión 12.01.14. Extraído el 2 de julio de 2012 de http://carlosreynoso.com.ar

Rodríguez, J. (2012). Análisis sobre la integración en el sistema educativo de las TIC: proyectos institucionales y formación permanente. Revista Teoría de la Educación: Educación y Cultura en la Sociedad de la Información.13(3), 129-144 [fecha de consulta 23/03/2013]. http://campus.usal.es/ revistas _trabajo/index.php/revistatesi/article/view/9134/9367

Satish, K.V. (2012). The Role of Teacher and Technology in Perspective of Classroom Teaching. Journal of Educational and Practice, 3(1), 49-54.

Scheiter, K. \& Gerjets, P. (2007). Learner control in Hypermedia Environments. Educational Psychology Review, 19, 285-307.

Spiro, R.J. \& Jenhng, J.C. (1990). Cognitive flexibility and hypertext: Theory and technology for the non-linear and multidimensional traversal of complex subject matter. En D. Nix, D. \& R. Spiro, R. (Eds.), Cognition, Education, and Multimedia (pp. 163-205). Hillsdale, NJ : Erlbaum.

Spiro, R.J. et al. (2007). Cognitive Flexibility, Constructivism, and Hipertext: Random Access Instruction for Advanced Knowledge Acquisition in Ill-Structured Domains [original publicado en 1992 en T. Duffy \& D. Jonassen (Eds.), 57-76]. $\begin{array}{lllllll}\text { Extraído el } & 25 & \text { de } & \text { junio } & \text { de } & 2012 & \text { de }\end{array}$ http://phoenix.sce.fct.unl.pt/simposio/Rand_Spiro.htm (1 de 23) 9/8/2007.

Para citar el presente artículo puede utilizar la siguiente referencia:

Argos, J. y Ezquerra, P. (2013). Entornos hipertextuales y educación. Revista Teoría de la Educación: Educación y Cultura en la Sociedad de la Información. 14(3), 175-190 [Fecha de consulta: dd/mm/aaaa].

http://campus.usal.es/ revistas_trabajo/index.php/revistatesi/article/view/11356/11773

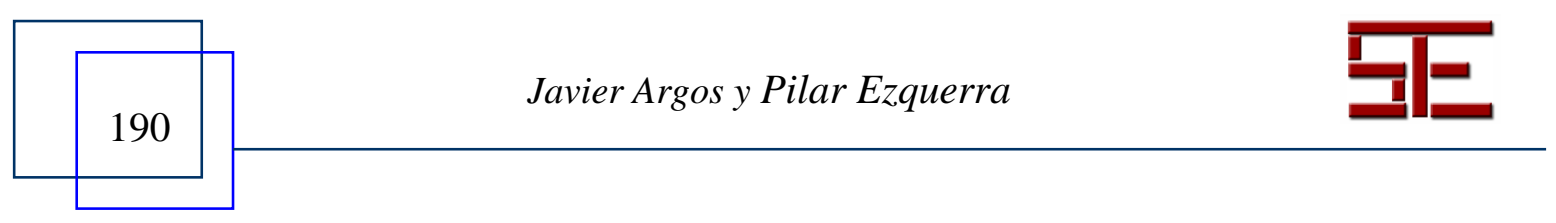

